\title{
A Genome Sequence Resource for the Geographically Widespread Anthracnose Pathogen Colletotrichum asianum
}

\author{
Yanan Meng, ${ }^{1}$ Yihua Ren, ${ }^{1}$ Wenjing Wang, ${ }^{1}$ Mark L. Gleason, ${ }^{2}$ Rong Zhang, ${ }^{1, \dagger}$ and Guangyu Sun ${ }^{1, \dagger}$ \\ ${ }^{1}$ State Key Laboratory of Crop Stress Biology in Arid Areas and College of Plant Protection, Northwest \\ A\&F University, Yangling, Shaanxi Province 712100, China \\ ${ }^{2}$ Department of Plant Pathology and Microbiology, lowa State University, Ames, IA 50011, U.S.A.
}

\begin{abstract}
Colletotrichum asianum is a worldwide plant pathogen causing serious fruit or leaf anthracnose diseases on a variety of plant hosts such as mango, coffee berry, chili, and other potential hosts, and it is distributed widely in Asia, America, Africa, and Oceania. This is the first genome resource available for $C$. asianum. The draft genome assembly will allow further analysis of species diversity and evolutionary mechanisms, and may serve as a foundation for genetic analysis that leads to greater understanding of interactions between plants and fungal pathogens.
\end{abstract}

The genus Colletotrichum includes a number of important plant pathogens that cause diseases on a variety of plants worldwide (Cannon et al. 2012). Colletotrichum asianum Prihast., L. Cai \& K. D. Hyde is a species in the $C$. gloeosporioides species complex (Prihastuti et al. 2009) and causes anthracnose diseases that may result in significant losses on several tropical fruit crops such as mango. The species was originally described as a coffee berry (Coffee arabica L.) pathogen from Thailand (Prihastuti et al. 2009). Later, the pathogen was also reported causing chili (Capsicum annuum L.) anthracnose (Phoulivong et al. 2010) and fruit or leaf anthracnose on mango (Mangifera indica L.) (Mo et al. 2018). Furthermore, potential hosts included papaya (Carica papaya L.), banana (Musa acuminata Colla), guava (Psidium guajava L.), bell pepper (Capsicum annuum L.), avocado (Persea americana Mill.), and tree tomato (Solanum betaceum Cav.) (Cabrera et al. 2018; Giblin et al. 2018; Lima et al. 2015). Colletotrichum asianum is geographically widespread, with a distribution that includes Asia, America, Africa, and Oceania (Krishnapillai and Wilson Wijeratnam 2014; Lima et al. 2013; Mo et al. 2018; Phoulivong et al. 2010; Sharma et al. 2013; Tovar-Pedraza et al. 2020; Weir et al. 2012; Zakaria et al. 2015). These studies indicate that $C$. asianum can adapt to diverse geographic environments and hosts. Sequencing the genome of the fungus may advance genetic understanding of the nature of host-pathogen interactions, assisting in the development of more effective disease control measures.

Colletotrichum asianum ICMP 18580 was deposited at the International Collection of Microorganisms from Plants (ICMP) in New Zealand as the ex-type strain (Weir et al. 2012). The fungus was originally isolated from asymptomatic coffee berry (Coffee arabica L.) at Chiang Mai, Thailand, and identified as a pathogen on mature fruit, causing typical anthracnose (Prihastuti et al. 2009). In the present study, this isolate was repurified using the single-spore isolation method. Highly purified total genomic DNA was extracted from fungal mycelia collected from 5-day-old single-spore cultures grown on potato dextrose agar at room temperature in darkness based on a modified cetyltrimethylammonium bromide procedure (Murray and Thompson 1980). Genomic library construction was accomplished by the

†Corresponding authors: G. Y. Sun; sgy@ nwsuaf.edu.cn; and R. Zhang; rongzh@ nwsuaf.edu.cn

The author(s) declare no conflict of interest.

Accepted for publication 2 March 2020.
Funding

This research was supported by the National Natural Science Foundation of China (31772113 and 31972220) and the Earmarked Fund for China Agriculture Research System (CARS-27).

\section{Keywords}

disease management, fruit, fungi, tropical plants, vegetables 
Table 1. Complete genome summary of Colletotrichum asianum strain ICMP 18580

\begin{tabular}{lc} 
Variables & Statistics \\
Sequence coverage & $167 \times$ \\
Assembly size & $64.73 \mathrm{Mb}$ \\
Number of scaffolds & 486 \\
Scaffold N & $38,703 \mathrm{bp}$ \\
GC content & $49.37 \%$ \\
Number of predicted genes & 17,966 \\
Candidate effectors & 351 \\
Repeat rate & $4.86 \%$ \\
BUSCO estimates & $99.65 \%$ \\
\hline
\end{tabular}

NEBNext Ultra II DNA Library Prep Kit for Illumina following instructions from the supplier, and genome sequencing was performed on the lllumina HiSeq2500 platform (Biomarker, Beijing, China). The mean insertion size of the sequencing library was $270 \mathrm{bp}$ and the sequencing strategy was 150-bp paired-ends reads. Trimmomatic v0.32 (Bolger et al. 2014) was used to remove adaptors and trim ends of the raw Illumina paired-end data, and the quality of trimmed Illumina reads was checked by FastQC v0.11.8 (https://www.bioinformatics.babraham.ac.uk/ projects/fastqc). In total, 73,136,620 reads were generated, with 167-fold coverage. Trimmed Illumina reads were assembled into scaffolds with ABySS assembler v1.3.5 (Simpson et al. 2009). To get the best assembly, the optimal kmer value 63 was empirically determined from 26 different kmer values (51 to 101 with step 2). Trimmed Illumina reads produced a final assembly comprising 1,105 contigs assembled into 486 scaffolds. The total length of all scaffolds was $64.73 \mathrm{Mb}\left(\mathrm{N}_{50}=389,703 \mathrm{bp}\right)$; summary statistics are provided in Table 1 . The assembly size is the largest of the published genomes in the Colletotrichum gloeosporioides species complex (Cannon et al. 2012), including C. gloeosporioides Cg-14 (53.21 Mb) (Alkan et al. 2013), C. fructicola 1104-7 (57.08 Mb) (Liang et al. 2018), and C. siamense ICMP 18578 (55.96 Mb) (Meng et al. 2019). The completeness of the assembly was assessed using BUSCO v1.2 with the lineage-specific profile library fungi_odb9 (Simão et al. 2015), which showed that the genome assembly contained $99.65 \%$ of the 1,438 conserved fungal orthologs. In order to predict the proportion of repeat sequences in the genome, a protocol combining two different strategies was applied. First, homology-based repeat element prediction was performed using Repeat Masker v4.0.5 (http://www.repeatmasker.org) with the RepBase fungal library (build on 20170127). Second, de novo repeats detection was performed using the Repeat Modeler v1.0.7 pipeline with default settings (Saha et al. 2008). A filtered custom RepeatModeler library was generated after removing repetitive gene sequences from the original RepeatModeler repeat library; then, repeat sequence prediction and categorizing were operated by RepeatMasker based on the filtered custom RepeatModeler library. Finally, the results of the above two strategies were combined and analyzed. About $4.86 \%$ of sequences were identified as repeat sequences. For contrast, we subjected the genomes of C. fructicola 1104-7 (Liang et al. 2018), C. gloeosporioides Cg-14 (Alkan et al. 2013), and C. siamense ICMP 18578 (Meng et al. 2019) to detect repeat sequences using the same protocol. The genomes of these species encompassed $2.09,1.26$, and $1.56 \%$ repeat sequences, respectively. The percentage of $C$. asianum ICMP 18580 genome repeat sequence was significantly higher than those of the other three species ( ${ }^{*}, P=0.006, t$ test). MAKER v2.31.8 (Holt and Yandell 2011) was utilized for gene prediction to integrate annotations from different ab initio gene prediction tools GeneMark-ES (Ter-Hovhannisyan et al. 2008) and Augustus v3.1 (Stanke and Morgenstern 2005) together with all protein sequences of homologous species, including C. graminicola M1.001 v1.0 (Cannon et al. 2012), C. gloeosporioides Cg-14 v1.0 (Alkan et al. 2013), C. fructicola Nara gc5 v1.0 (Gan et al. 2013), and C. fioriniae PJ7 v1.0 (Baroncelli et al. 2014), which belong to the same genus with C. asianum. GeneMark-ES was self-trained and the gene models of the Glomerella cingulata 23 strain (Weir et al. 2012) obtained from the Joint Genome Institute (https://genome.jgi. doe.gov/programs/fungi/index.jsf) were used to train Augustus. Overall, 17,966 proteincoding gene models were predicted from the $C$. asianum genome assembly. Of the protein-coding gene models, $60.14 \%(10,805)$ contained PFAM domains annotated by InterProScan 5.2-45.0 (threshold e-value of 1E-05) (Jones et al. 2014). Meanwhile 2,353 proteins were predicted with signal peptide by using SignalP v4.1 (Petersen et al. 2011). 
Among the 2,353 proteins, 1,718 were computationally predicted to localize in the extracellular space as secreted proteins based on results from WoLF PSort v0.2 (Horton et al. 2007). Within the predicted secretome, in total, 712 small secreted proteins (SSPs) were predicted. Candidate SSPs were defined here as secreted proteins that were smaller than 300 amino acids (Mirzadi Gohari et al. 2015). Among the predicted SSPs, 351 cysteine-rich small secreted proteins (the percentage of cysteine residues in the protein was at least twice as high as the average percentage of cysteine residues in all predicted proteins of this species) were identified, which is generally considered as evidence that a protein is a candidate-secreted effector (Ohm et al. 2012). In all, 1,106 carbohydrate active enzymes (CAZymes) were predicted from the predicted proteomes by dbCAN database 2.0 (threshold e-value of 1E-3) (Yin et al. 2012). In fungi, plant cell-wall-degrading enzymes (PCWDEs) are known to play important roles in plant pathogenesis (Ospina-Giraldo et al. 2003; Walton 1994). Cellulases, hemicellulases, and pectinases together formed PCWDEs that we considered in present study. These three types of PCWDEs are distributed in different CAZyme families and belong to three functional classes (i.e., carbohydrate esterases, glycoside hydrolases, and polysaccharide lyases). Thus, we could pick out these PCWDE-involved families from the predicted CAZymes and regard them as putative PCWDEs. Finally, of the 1,106 predicted CAZymes, 368 were predicted to be putative PCWDEs (Gan et al. 2013). The draft assembly will allow further analysis of the pathogen species and population diversity and evolutionary mechanism, and may serve as a foundation for genetic analysis that leads to greater understanding of interactions between plants and fungal pathogens.

The genome assembly of $C$. asianum ICMP 18580 was deposited at GenBank with the accession number WOWK00000000 (BioSample: SAMN13475008). The version described in this article is version WOWK01000000.

\section{Acknowledgments}

We thank the reviewers for their valuable suggestions on the original manuscript and L. Cai, Institute of Microbiology, Chinese Academy of Sciences, for kindly providing the type strain.

\section{Literature Cited}

Alkan, N., Meng, X. C., Friedlander, G., Reuveni, E., Sukno, S., Sherman, A., Thon, M., Fluhr, R., and Prusky, D. 2013. Global aspects of pacC regulation of pathogenicity genes in Colletotrichum gloeosporioides as revealed by transcriptome analysis. Mol. Plant-Microbe Interact. 26:1345-1358.

Baroncelli, R., Sreenivasaprasad, S., Sukno, S. A., Thon, M. R., and Holub, E. 2014. Draft genome sequence of Colletotrichum acutatum sensu lato (Colletotrichum fioriniae). Genome Announce. 2:e00112-14.

Bolger, A. M., Lohse, M., and Usadel, B. 2014. Trimmomatic: A flexible trimmer for Illumina sequence data. Bioinformatics 30:2114-2120.

Cabrera, L., Rojas, P., Rojas, S., Pardo-De la Hoz, C. J., Mideros, M. F., Danies, G., Lopez-Kleine, L., Jimenez, P., and Restrepo, S. 2018. Most Colletotrichum species associated with tree tomato (Solanum betaceum) and mango (Mangifera indica) crops are not host-specific. Plant Pathol. 67:1022-1030.

Cannon, P. F., Damm, U., Johnston, P. R., and Weir, B. S. 2012. Colletotrichum —Current status and future directions. Stud. Mycol. 73:181-213.

Gan, P., Ikeda, K., Irieda, H., Narusaka, M., O'Connell, R. J., Narusaka, Y., Takano, Y., Kubo, Y., and Shirasu, K. 2013. Comparative genomic and transcriptomic analyses reveal the hemibiotrophic stage shift of Colletotrichum fungi. New Phytol. 197:1236-1249.

Giblin, F. R., Tan, Y. P., Mitchell, R., Coates, L. M., Irwin, J. A. G., and Shivas, R. G. 2018. Colletotrichum species associated with pre-and post-harvest diseases of avocado and mango in eastern Australia. Australas. Plant Pathol. 47:269-276.

Holt, C., and Yandell, M. 2011. MAKER2: An annotation pipeline and genomedatabase management tool for second-generation genome projects. BMC Bioinf. 12:491.

Horton, P., Park, K. J., Obayashi, T., Fujita, N., Harada, H., Adams-Collier, C. J., and Nakai, K. 2007. WoLF PSORT: Protein localization predictor. Nucleic Acids Res. 35:W585-W587.

Jones, P., Binns, D., Chang, H. Y., Fraser, M., Li, W., McAnulla, C., McWilliam, H., Maslen, J., Mitchell, A., Nuka, G., Pesseat, S., Quinn, A. F., Sangrador-Vegas,
A., Scheremetjew, M., Yong, S. Y., Lopez, R., and Hunter, S. 2014. InterProScan 5: Genome-scale protein function classification. Bioinformatics 30:1236-1240. Krishnapillai, N., and Wilson Wijeratnam, R. S. 2014. First report of Colletotrichum asianum causing anthracnose on Willard mangoes in Sri Lanka. New Dis. Rep. 29:1.

Liang, X. F., Wang, B., Dong, Q. Y., Li, L. N., Rollins, J. A., Zhang, R., and Sun, G. Y. 2018. Pathogenic adaptations of Colletotrichum fungi revealed by genome wide gene family evolutionary analyses. PLoS One 13:e0196303.

Lima, N. B., Batista, M. V. de A., De Morais, M. A., Jr., Barbosa, M. A. G., Michereff, S. J., Hyde, K. D., and Câmara, M. P. S. 2013. Five Colletotrichum species are responsible for mango anthracnose in northeastern Brazil. Fungal Divers. 61: 75-88.

Lima, N. B., Lima, W. G., Tovar-Pedraza, J. M., Michereff, S. J., and Câmara, M. P. S. 2015. Comparative epidemiology of Colletotrichum species from mango in northeastern Brazil. Eur. J. Plant Pathol. 141:679-688.

Meng, Y. N., Gleason, M. L., Zhang, R., and Sun, G. Y. 2019. Genome sequence resource of the wide-host-range anthracnose pathogen Colletotrichum siamense. Mol. Plant-Microbe Interact. 32:931-934.

Mirzadi Gohari, A., Ware, S. B., Wittenberg, A. H. J., Mehrabi, R., Ben M'Barek, S., Verstappen, E. C. P., van der Lee, T. A. J., Robert, O., Schouten, H. J., De Wit, P. P. J. G. M., and Kema, G. H. J. 2015. Effector discovery in the fungal wheat pathogen Zymoseptoria tritici. Mol. Plant Pathol. 16:931-945.

Mo, J. Y., Zhao, G., Li, Q. L., Solangi, G. S., Tang, L. H., Guo, T. X., Huang, S. P., and Hsiang, T. 2018. Identification and characterization of Colletotrichum species associated with mango anthracnose in Guangxi, China. Plant Dis. 102: 1283-1289.

Murray, M. G., and Thompson, W. F. 1980. Rapid isolation of high molecular weight plant DNA. Nucleic Acids Res. 8:4321-4326.

Ohm, R. A., Feau, N., Henrissat, B., Schoch, C. L., Horwitz, B. A., Barry, K. W., Condon, B. J., Copeland, A. C., Dhillon, B., Glaser, F., Hesse, C. N., Kosti, I., LaButti, K., Lindquist, E. A., Lucas, S., Salamov, A. A., Bradshaw, R. E., Ciuffetti, 
L., Hamelin, R. C., Kema, G. H., Lawrence, C., Scott, J. A., Spatafora, J. W., Turgeon, B. G., de Wit, P. J., Zhong, S., Goodwin, S. B., and Grigoriev, I. V. 2012. Diverse lifestyles and strategies of plant pathogenesis encoded in the genomes of eighteen Dothideomycetes fungi. PLoS Pathog. 8:e1003037.

Ospina-Giraldo, M. D., Mullins, E., and Kang, S. 2003. Loss of function of the Fusarium oxysporum SNF1 gene reduces virulence on cabbage and Arabidopsis. Curr. Genet. 44:49-57.

Petersen, T. N., Brunak, S., von Heijne, G., and Nielsen, H. 2011. SignalP 4.0: Discriminating signal peptides from transmembrane regions. Nat. Methods 8:785-786.

Phoulivong, S., Cai, L., Chen, H., McKenzie, E. H. C., Abdelsalam, K., Chukeatirote, E., and Hyde, K. D. 2010. Colletotrichum gloeosporioides is not a common pathogen on tropical fruits. Fungal Divers. 44:33-43.

Prihastuti, H., Cai, L., Chen, H., McKenzie, E. H. C., and Hyde, K. D. 2009. Characterization of Colletotrichum species associated with coffee berries in northern Thailand. Fungal Divers. 39:89-109.

Saha, S., Bridges, S., Magbanua, Z. V., and Peterson, D. G. 2008. Empirical comparison of ab initio repeat finding programs. Nucleic Acids Res. 36:2284-2294.

Sharma, G., Kumar, N., Weir, B. S., Hyde, K. D., and Shenoy, B. D. 2013. The ApMat marker can resolve Colletotrichum species: A case study with Mangifera indica. Fungal Divers. 61:117-138.

Simão, F. A., Waterhouse, R. M., loannidis, P., Kriventseva, E. V., and Zdobnov, E. M. 2015. BUSCO: Assessing genome assembly and annotation completeness with single-copy orthologs. Bioinformatics 31:3210-3212.
Simpson, J. T., Wong, K., Jackman, S. D., Schein, J. E., Jones, S. J. M., and Birol, I. 2009. ABySS: A parallel assembler for short read sequence data. Genome Res. 19:1117-1123.

Stanke, M., and Morgenstern, B. 2005. AUGUSTUS: A web server for gene prediction in eukaryotes that allows user-defined constraints. Nucleic Acids Res. 33:W465-W467.

Ter-Hovhannisyan, V., Lomsadze, A., Chernoff, Y. O., and Borodovsky, M. 2008. Gene prediction in novel fungal genomes using an ab initio algorithm with unsupervised training. Genome Res. 18:1979-1990.

Tovar-Pedraza, J. M., Mora-Aguilera, J. A., Nava-Diaz, C., Lima, N. B., Michereff, S. J., Sandoval-Islas, J. S., Câmara, M. P. S., Teliz-Ortiz, D., and Leyva-Mir, S. G. 2020. Distribution and pathogenicity of Colletotrichum species associated with mango anthracnose in Mexico. Plant Dis. 104: 137-146.

Walton, J. D. 1994. Deconstructing the cell wall. Plant Physiol. 104:1113-1118.

Weir, B. S., Johnston, P. R., and Damm, U. 2012. The Colletotrichum gloeosporioides species complex. Stud. Mycol. 73:115-180.

Yin, Y. B., Mao, X. Z., Yang, J. C., Chen, X., Mao, F. L., and Xu, Y. 2012. dbCAN: A web resource for automated carbohydrate-active enzyme annotation. Nucleic Acids Res. 40:W445-W451.

Zakaria, L., Juhari, N. Z., Vijaya, S. I., and Anuar, I. S. M. 2015. Molecular characterization of Colletotrichum isolates associated with anthracnose of mango fruit. Sains Malays. 44:651-656. 